\title{
Erratum: Spontaneous magnetization and electron momentum density in three-dimensional quantum dots [Phys. Rev. B 68, 165326 (2003)]
}

R. Saniz, B. Barbiellini, A. B. Denison, and A. Bansil (Received 9 September 2011; published 27 September 2011)

DOI: 10.1103/PhysRevB.84.119907

The Hamiltonian in Eq. (1) contains a typographical error; it should read

$$
\hat{H}=\sum_{\nu \sigma} \epsilon_{\nu}^{0} a_{\nu \sigma}^{\dagger} a_{\nu \sigma}+\frac{1}{2} U \sum_{\nu \nu^{\prime} \sigma} a_{\nu \sigma}^{\dagger} a_{\nu^{\prime}-\sigma}^{\dagger} a_{\nu^{\prime}-\sigma} a_{\nu \sigma} .
$$

In our work, of course, we used this correct Hamiltonian. Thus, none of our results are affected in any way by the misprint.

*Present address: Departement Fysica, Universiteit Antwerpen, Groenenborgerlaan 171, B-2020 Antwerpen, Belgium. 